\title{
Baculoviral Co-Expression of HA, NA and M1 Proteins of Highly Pathogenic H5N1 Influenza Virus in Insect Cells
}

\author{
Farida Behzadian ${ }^{1}$, Zahra Goodarzi ${ }^{2}$, Fatemeh Fotouhi ${ }^{3}$, Esmaeil Saberfar ${ }^{2, *}$

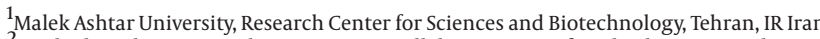 \\ ${ }_{3}^{2}$ Applied Virology Research Center, Baqiyatallah University of Medical Sciences, Tehran, IR Iran \\ ${ }^{3}$ Influenza Research Labs, Pasteur Institute of Iran, Tehran, IR Iran \\ *Corresponding author: Esmaeil Saberfar, Department of Applied Virology, Baqiyatallah University of Medical Sciences, Tehran, IR Iran. Tel: +98-2188211523, Fax:+98-2188211523, E-mail: \\ fbehzadian@yahoo.com
}

Received: August 22, 2012; Revised: December 12, 2012; Accepted: January 12, 2013

Background: Highly pathogenic H5N1 influenza virus is causing damages to the poultry industry and is responsible for loss of human lives. Vaccination is the most effective method to prevent and control influenza infections. Recombinant virus-like particles (VLPs) by baculovirus expression vector system have been suggested as a promising platform for new viral vaccines.

Objectives: In this study we constructed a recombinant baculovirus that was potent to express influenza structural proteins; Haemagglutinin (HA) and Neuraminidase (NA) as well as matrix protein (M1) which is essential to generate immunogenic VLPs in insect cells.

Materials and Methods: Atriplet cassette providing simultaneous and independent expression of HA, NA and M1 genes of avian influenza virus (A/Indonesia/5/05(H5N1)) was designed and subjected to synthesis. The cassette was then cloned into pFastBac1 plasmid and then transformed in to competent Escherichia coli DH10Bac cells and the recombinant bacmids were produced following the homologous Tn7 site-specific transposition. This construction was verified by polymerase chain reaction (PCR) and then transfected into Sfg insect cells to package new recombinant baculovirus expressing complex proteins of the interest.

Results: Restriction map of pFastBacIHNM1 plasmid confirmed the fidelity of the clone. The PCR carried out on the recombinant bacmids as template indicated that a proper homologous recombination has occurred between pFastBacIHNM1 donor plasmid and the bacmid. Following the transfection of new recombinant bacmids to Sf9 cells, cytopathic effects were observed which indicating the successful packaging of recombinant baculovirus. Protein analysis of the infected Sf9 cells showed that all target proteins were efficiently expressed at the same time.

Conclusions: The recombinant baculovirus constructed in this work has proper characteristics to produce H5N1 influenza virus-like particles in Sf9 cells.

Keywords: H5 N1 Influenza Virus; Baculovirus; Recombinant Bacmid; Insect Cell

\section{Background}

Influenza A virus, a member of Orthomyxoviridae family, is able to infect a large variety of animals as well as humans although aquatic birds are the natural sources (1). The associated clinical signs in hosts and the outcome of influenza A virus infections considerably vary with the species of the host and strain of virus. Influenza A viruses that are segmented negative strand genome viruses, classified according to the serological subtypes of the viral surface proteins, the hemagglutinin (HA) and neuraminidase (NA). The HA has 16 subtypes (H1 - H16) containing neutralizing epitopes and there are nine neuraminidase (N1 - N9) or "N" subtypes. The "H" and "N" subtypes are able to assort into any possible combination. Many of these combinations have been found in birds as main natural reservoir species, although some combinations are more common than others. To date, only viruses with $\mathrm{H} 5, \mathrm{H} 7$ and $\mathrm{H} 10$ subtypes have contributed to highly pathogenic avian influenza in susceptible species, but not all $\mathrm{H} 5, \mathrm{H} 7$ and $\mathrm{H} 10$ viruses are sever $(2,3)$.
H5N1 virus has long been known as a veterinary pathogen but it re-emerged for the first time in Southeast Asia around 1997. Until 2003, this strain was relatively circulating and had been mainly involved in sporadic outbreaks. In 2004, the infection incidence in poultry increased in Asia and as fatal cases of human infections in several Southeast Asian countries were confirmed. By 2006, the virus had further spread to Africa and Europe, and as far west as the United Kingdom (4). The prevalence of avian H5N1 influenza A infections in humans has not been definitively determined. In February 2012, 602 H5N1 infection cases in humans with an overall fatality rate of $>50 \%$ were confirmed by the world health organization (WHO). Moreover, some isolates have shown resistance to the antiviral drugs Amantadine and Rimantadine $(5,6)$. Due to vast global spread of the infection and its ability to adapt to mammalian hosts which resulted in acquiring the genetic changes for efficient human- to- human transmission, H5N1 viruses have been considered as avian strains with pandemic potential risk $(1,6)$. 
Considering the influenza epidemics or pandemics, the vaccination is the most effective method to prevent and control influenza infections $(7,8)$. Currently, two types of licensed influenza vaccines are mainly used, chemically inactivated consisted of detergent-split virion subunits, and live attenuated vaccine. Both vaccines are produced, using embryonated chicken eggs as substrates which have several drawbacks including local or systemic allergic reactions to egg-derived vaccine components and disruption the supply of eggs for vaccine manufacturing due to avian influenza virus outbreak. Moreover, both kinds of vaccines provide a short duration of immune responses that are not completely effective, particularly in the elderly people (9).

To overcome the mentioned challenges, molecular biotechnologists have introduced novel lines to produce more safe and effective vaccines $(10,11)$. One of these lines, offering certain advantages over traditional influenza vaccine technologies, is the recombinant virus-like particles (VLPs) which are considered a promising eggindependent vaccine approach. Influenza VLPs do not require handling of live pathogenic virus during the development, manufacturing or administration $(12,13)$. Influenza VLPs have been generated from various strains of virus including H1N1, H3N2, H5N1, H9N2, 1918 pandemic virus and 2009 pandemic virus in insect cell system. These VLPs composing from four, three or even two envelope proteins that all are highly-immunogenic and could provide effective protection against influenza virus infection in animal models without any adjuvant. Because of significant role of the matrix protein (M1) in influenza VLP formation and budding process, it has been used as a basic component in generation of all influenza VLPs (14).

\section{Objectives}

In this study, we constructed a new recombinant poly- cistronic baculovirus which is basically required for influenza VLP production in insect cells by using Bac-to-Bac system. This new recombinant baculovirus, simultaneously and independently expresses the three main components of influenza VLP including hemagglutinin (HA), neuraminidase (NA) and matrix (M1) proteins which are specific for highly pathogenic $\mathrm{H} 5 \mathrm{~N} 1$ avian influenza virus.

\section{Materials and Methods}

\subsection{Cassette Designing and Cloning Strategy}

A cassette consisted of HA, NA and M1 sequences of highly pathogenic influenza virus (A/Indonesia/5/05(H5N1), regulatory genetic elements such as promoters and polyadenylation signals, as well as spacer sequences containing appropriate restriction sites regarding the cloning scheme of full "HNM1 cassette" was designed and thoroughly synthesised (Genescript, China). As demonstrated in Figure 1, from 5' to 3', the first open reading frame (ORF) is HA (1704 bp) that following the cloning of HNM1 cassette will place downstream of polyhidrin (polh) promoter belongs to pFastBacI donor plasmid. There is a SV40 polyadenylation sequence after HA ORF which improves the level of expression. Then the NA transcriptional unit has designed which starts with polh promoter sequence followed by the NA ORF (1407 bp) and SV40 polyadenylation sequences. Behind this unite there is the next sequence of polh promoter and M1 ORF in its downstream. After the cloning of cassette into pFastBacI plasmid, the plasmid SV40 polyadenylation signal will position in downstream of M1 ORF (756bp) and constitute the M1 transcriptional unit.

The HNM1 cassette (4622 bp) was cut by EcoRI/SphI double digestion and cloned into pFastBacI through the same restriction sites to construct "pFastBacIHNM1 "donor plasmid.

Figure 1. Schematic Diagram of HNM1 Full Cassette

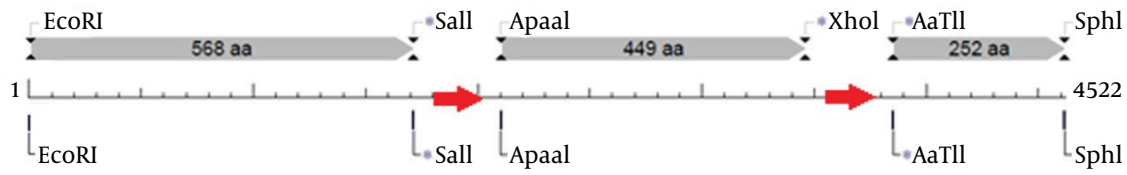

The position of three independent transcriptional unites, polyhidrine promoters (red arrows) and crucial restriction sites have been shown in the diagram drawn by NEBcutter online software (New England Biolab).

\subsection{Generation of Recombinant Bacmid}

The recombinant pFastBacIHNM1 donor plasmid was transformed into the Escherichia coli DH10Bac competent cells for Tn7 site-specific transposition of the HNM1 cassette fragment to a bacmid DNA through lacZ gene disruption. The transformed cells were plated onto the LB agar containing Kanamycin $50 \mu \mathrm{g} / \mathrm{mL}$, Gentamicin
$7 \mu \mathrm{g} / \mathrm{mL}$, Tetracycline $10 \mu \mathrm{g} / \mathrm{mL}$, Bluo-gal $100 \mu \mathrm{g} / \mathrm{mL}$ and Isopropylthio- $\beta$-Galactoside (IPTG) $40 \mu \mathrm{g} / \mathrm{mL}$ and incubated at $37^{\circ} \mathrm{C}$ for 48 hours. The high-molecular-weight bacmid DNA was isolated from the overnight cultures by alkaline lysis purification according to the instructions supplied by the manufacturer (Invitrogen, USA).

Successful transposition was verified by PCR analysis using either M13/pUC or specific primers for M1 region with 
the following sequences: M13/pUC forward, 5'-CCCAGTCACGACGTTGTAAAACG-3', M13/pUC reverse, 5'-AGCGGATAACAATTTCACACAGG-3' and M1 forward, 5'-ATGAGTCTTCTA ACCGAGGTC-3'. Amplification reactions were performed under the following condition: 3 minutes at $95^{\circ} \mathrm{C}$ followed by 35 cycles of 1 minute at $95^{\circ} \mathrm{C}$ for denaturation, 1 minute at $58^{\circ} \mathrm{C}$ for primer annealing and 4 minutes at $68^{\circ} \mathrm{C}$ for extension, concluded by a final extension step at $68^{\circ} \mathrm{C}$ for 10 minutes. Dream Taq ${ }^{\mathrm{TM}}$ DNA Polymerase (Fermantas, Lithuania) optimized for robust amplifications on long targets was used in PCR mixture.

\subsection{Cell Culture and Transfection to Generate Re- combinant Baculovirus}

Spodoptra Frugipdera (Sf9) cells as adherent culture was purchased from the Cell Bank (Pasture Institute of Iran) and grown in Grace's medium supplemented with 10\% fetal bovine serum (Gibco, USA). Sf9 cells were transfected with the isolated recombinant DNA using Cellfectin (Invitrogen, USA) for the generation of the recombinant baculovirus according to the manufacturer instruction. The transfected cells were incubated at $27^{\circ} \mathrm{C}$ for $72-96$ hours, to allow baculovirus assembly and release into the culture media. Recombinant baculovirus production was monitored daily by visualization of the cytopathic effects. The culture medium was collected, clarified by low-speed centrifugation for 10 minutes at $1000 \mathrm{rpm}$ and subjected to multiple rounds of virus propagation.

\subsection{Expression and Determination of Recombi- nant Proteins}

The overnight cultured Sf9 cells were inoculated with recombinant baculovirus at a multiplicity of infection (MOI) of 10 and incubated at $27^{\circ} \mathrm{C}$ for 72 hours. The culture medium (supernatant) and the cells (pellet) were collected after centrifugation. To prepare cell lysate, the pelleted cells were washed three times with cold PBS, suspended in sample buffer (50 mMTris- $\mathrm{HCl}, \mathrm{pH} 8.5,5 \mathrm{mM}$ 2-mercaptoethanol, $100 \mathrm{mM} \mathrm{KCl,1} \mathrm{mM} \mathrm{PMSF)} \mathrm{and} \mathrm{sonicat-}$ ed three times for 10 seconds with 3 minute-intervals, at $50 \%$ power using ultrasonicator (UP $400 \mathrm{~s}$ ). After centrifugation at $12000 \mathrm{rpm}$ for 15 minutes at $4^{\circ} \mathrm{C}$, the clarified supernatant was stored at $-20^{\circ} \mathrm{C}$ for further analysis. Uninfected Sf9 cells which were similarly treated were used as a negative control.

The protein samples were examined by SDS-PAGE and Coomassie staining (15). The separated proteins on SDSPAGE were also transferred to nitrocellulose membranes using semi-dry electroblot system (BioRad, Germany) according to the standard protocols. Briefly, the membranes were blocked with $1 \%$ bovine serum albumin. The blotted proteins were separately reacted with mouse anti-HA (ProSci, USA), anti-NA (AbCam, USA) and anti- M1 (SeroTec, UK) monoclonal antibodies diluted 1:200 in TBST for 2 hours at room temperature. The appropriate secondary antibody, goat anti-mouse IgG conjugated with
HRP (Razi Fara Teb, Iran) in 1:2000 dilutions, was used as secondary antibody. The protein bounds were developed by staining the membranes with diaminobenzidine (16).

\section{Results}

\subsection{Construction of the Recombinant Bacmid DNA}

Following the transformation of DH10Bac cells using pFastBacIHNM1 donor plasmids, the colonies harboring the recombinant bacmid were selected through blue and white screening. The white selected colonies were streaked again on complete medium of LB agar to certify the true phenotype.

Since restriction analysis is difficult to perform with high-molecular weight bacmid DNA, PCR was carried out to verify the presence of "HNM1 cassette" in the recombinant bacmid. The bacmid shuttle vectors containing M13/ pUC forward and reverse hybridization sites flanking the mini-att Tn7 sites within the LacZ region. With respect to position and length of inserted "HNM1 cassette" into bacmid, the $7200 \mathrm{bp}$ and $1400 \mathrm{bp}$ regions were amplify using M13/pUC specific primers and M1-forward primer along with M13/pUC-reverse primer in PCR mixtures, respectively (Figure 2).

Figure 2. Gel Electrophoresis of PCR Products Carried HNM1 Recombinant Bacmid

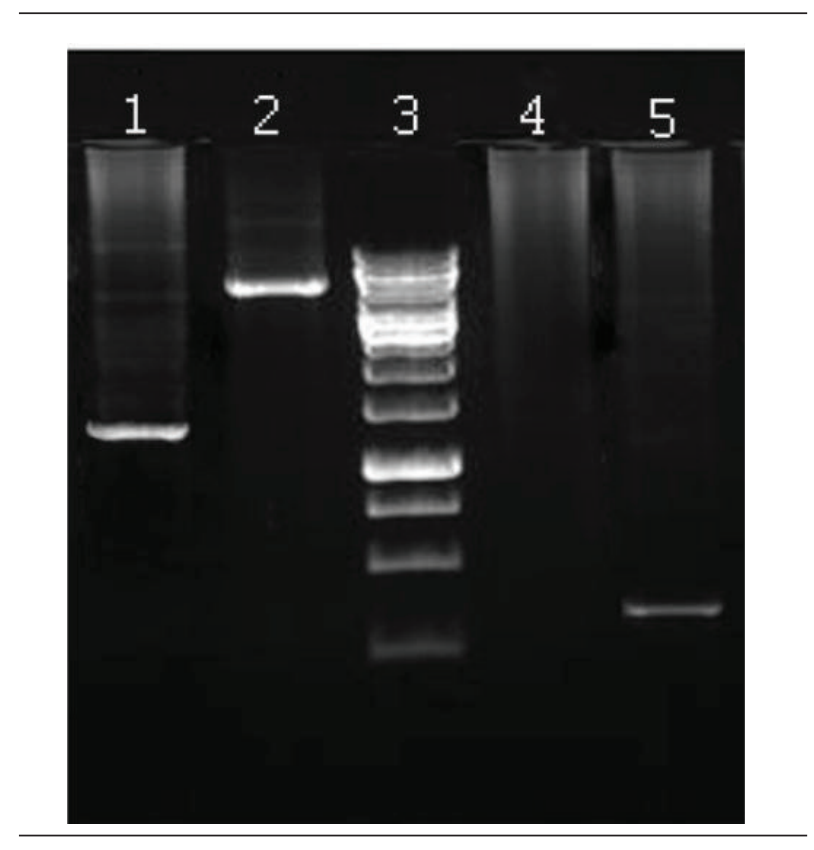

Lane 1: PCR product using M1 forward and M13 reverse primers on recombinant bacmid; amplification of a region with $1400 \mathrm{bp}$ in length as expected. Lane 2: PCR product using forward and reverse M13 primers on recombinant bacmid (7200bp). Lane 3:1Kb DNA marker (Fermentas). Lane 4: PCR product using M1 forward and M13 reverse primers on nonrecombinant bacmid as a negative control. No band was observed as expected. Lane 5: PCR product using M13 primers (303bp) on nonrecombinant bacmid as a negative control. 


\subsection{Transfection of Sfg Cells}

The isolated recombinant bacmid DNA were transfected to Sf9 cells using Cellfectin (Invitrogen). The transfected cells displayed typical cytopathic effects, i.e. low cell density, division stop, enlarged cells and poor adherence to the substrate, indicating that the virus production was taking place. The mock-transfected Sf9 cultures continued to divide and form a confluent normal cell monolayer (Figure 3).

Figure 3. The Mock-Transfected Sf9 Cultures
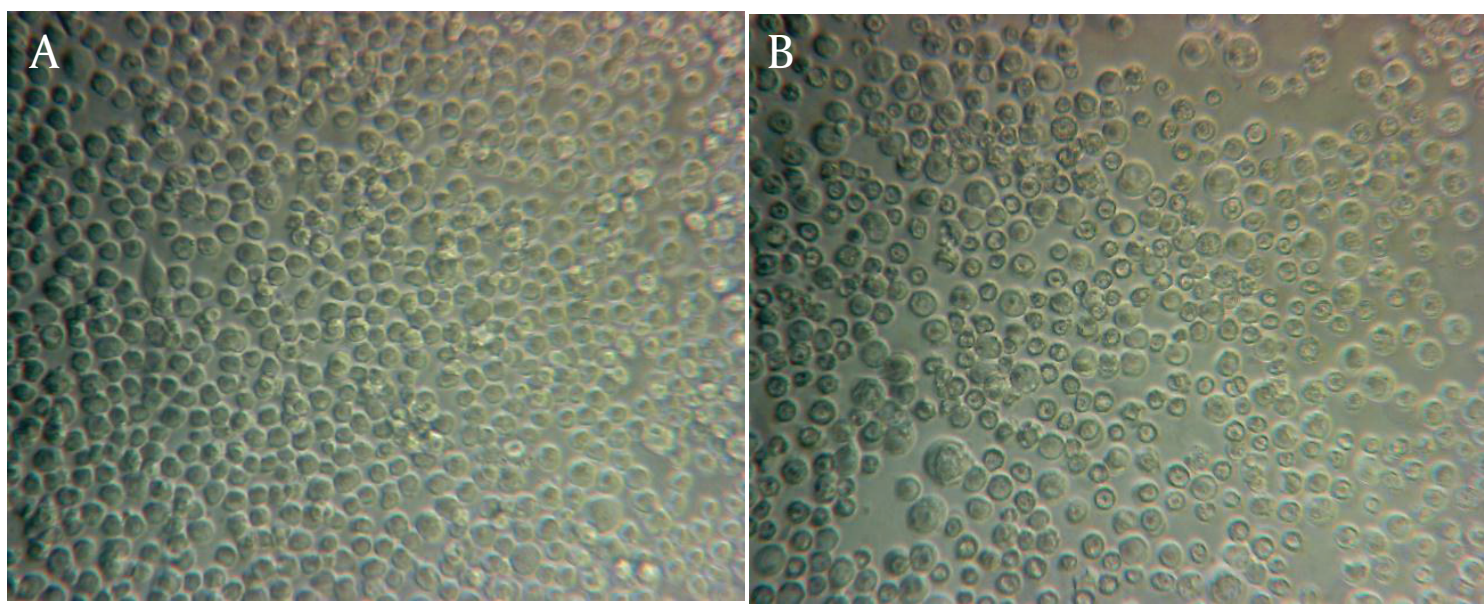

A, Mock-transfected Sf9 are the cells transfected without DNA to control potential effect of transfection reagent on the cells (negative control). B, Transfected Sf9 cells with recombinant bacmid DNA showing typical cytopathic effects resulted from recombinant baculovirus assembly and propagation ( $\times$ 200).

Figure 4. SDS-PAGE Analysis of Protein Extracted From Sf9 Cells Infected by Triple Baculovirus Recombinants

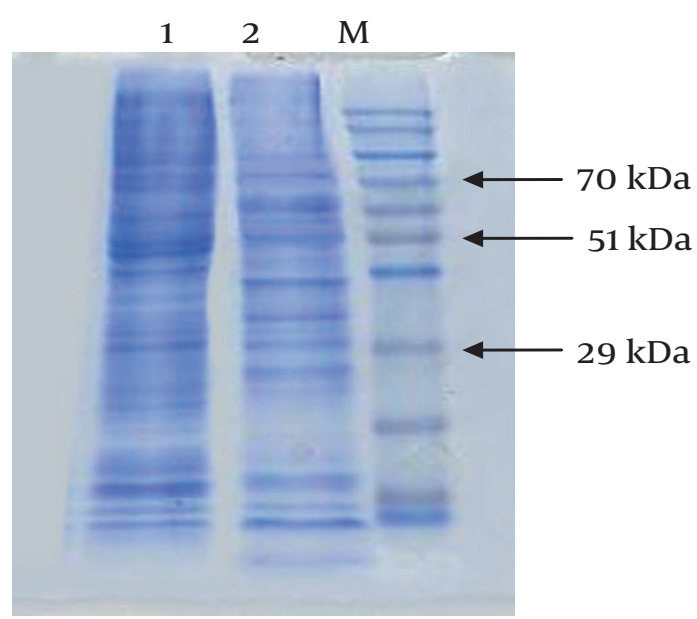

Lane 1, Uninfected Sf9 cells as control. Lane2, Lane M is protein marker (Vivantis).

\subsection{Determination of Recombinant Proteins}

To analyze the co-expression of three recombinant proteins, the Sf9 cells were infected with recombinant triple baculovirus and harvested after 72 hours. Total protein of the cell lysates was electrophoresed on $4-12 \%$ polyacrylamide gel SDS-PAGE. As shown in Figure 4 the exact polypeptide bands with predicted molecular weight of approximately 70,55 , and $27 \mathrm{kDa}$ representing the HA, NA and M1 respectively were detected. Western blot analysis of separated proteins on SDS-PAGE, probed with anti-HA, anti-NA and anti-M1 monoclonal antibodies proved the specificity of the detected bands and also demonstrated simultaneous and independent expression of the new recombinant influenza virus proteins (Figure 5). However analysis of the recombinant infected cell medium showed no detectable band. 
Figure 5. Western Blot Analysis of the Influenza Virus Proteins Expressed in Sf9 Cells Infected by Triple Baculovirus Recombinant

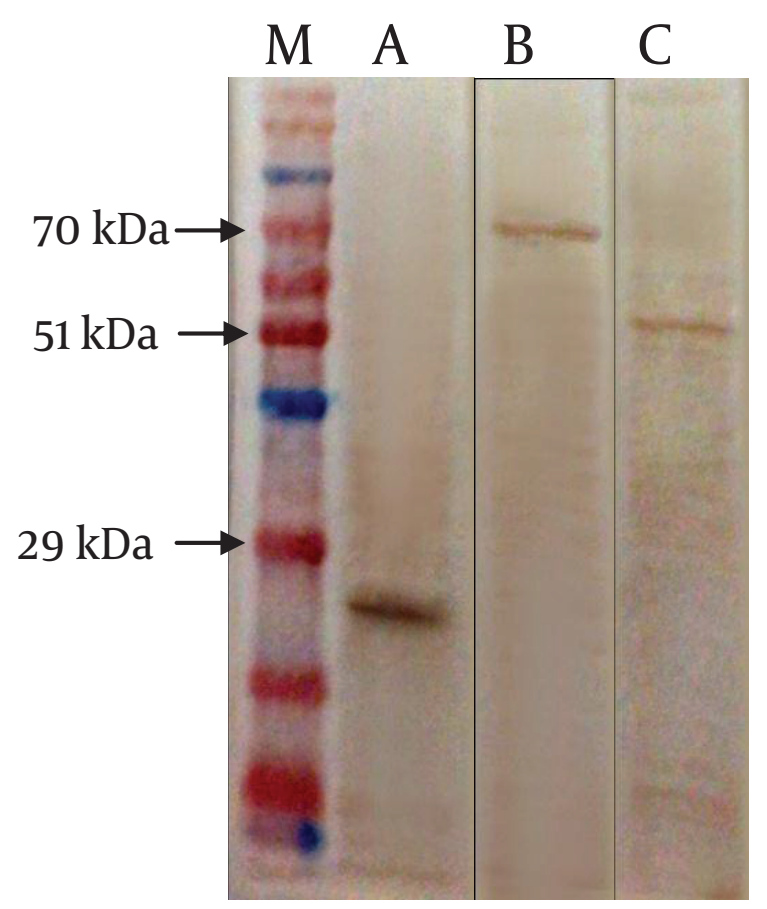

A, Expression of the M1 protein; B, Expression of the HA protein; C, Expression of the NA protein; M, protein marker (Vivantis).

\section{Discussion}

The production of Influenza VLPs as a vaccine candidate in insect expression system offers several advantages over current egg-based methods, particularly in terms of handling, robustness, yield, safety and cost efficiency (14). In this study, we designed and constructed the polycistronic baculovirus expressing envelope proteins of Influenza virus which is an essential need for influenza VLP production in insect cell lines.

Since the H5N1 avian influenza subtype apparently caused zoonotic infections and currently is endemic in vast areas of the world, we used HA, NA and M1 genomic sequences of this subtype to construct specific recombinant baculovirus that is required for H5N1 VLP production in insect cell system. The baculovirus expression vector system (BEVS) has several features such as it ease to design and construct new gene combinations, the scalability offered by the system, and the possibility of rapid a VLPvaccine production in an emerging situation (17-19). Several groups have reported the production of influenza VLPs using baculovirus expression vector in the insect cell system (20-23). They used four, three or even two viral structural protein genes to make immunogenic VLPs specific to different influenza subtypes (24). In addition different expression strategies have been used to produce proteins complexes in Baculoviral expression system (BVES) $(18,24)$. In some researches, the proteins are expressed from infection of multiple monocistronic baculoviruses (co-infection) $(25,26)$ while the others have exploited the use of a single polycistronic baculovirus to generate self-assembling multi-protein complexes (coexpression) $(11,27,28)$.

Pushko et al. found that only the co-expression of tricistronic baculoviruses led to the production of VLPS (24), and their results were termed "inconclusive" from co- infection experiments. Prel et al. decided to use polycristronic baculoviruses in their VLP studies, too $(11,28)$. Additionally, many have used polycristronic baculoviruses as a method of overcoming limitations inherent to co-infection, specifically the uneven distribution of virus taken up by cells. In other word, all essential proteins necessary for the generation of the recombinant product are expressed in the same infected cell by using polycistronic baculoviruses (24). According to the mentioned findings and points of view, in this study we designed a compound cassette comprising of three transcriptional unites to generate a recombinant tricistronic baculovirus.

Polh and p10 promoters both are used conventionally to generate large quantities of proteins in BVES. The effective performance of polh promoter to drive the coexpression of two or more genes of influenza virus in a single baculovirus has been previously shown (24-26). On the other hand, to avoid "competition effect" which may occurs when two genes expressed at the same time and at high level, under the control of two different promoters, in the HNM1 cassette, polh promoter has been choose for all transcription unites.

SV40 poly-A signal element is widely used in commercial vectors to drive transcriptional termination and improves translational efficiency of inserted genes. In the HNM1cassette, we also used SV40 poly-A signal sequence at the end of each HA and NA transcription unites but the third unite, M1, exploited the SV40 polyadenylation signal belongs to pFastBacI that following the insertion of cassette has located immediately downstream of M1 ORF. The protein molecular weight of A/Indonesia/5/05 HA and NA were 62.5 and $49.5 \mathrm{kDa}$ respectively. Nevertheless, the HA band in the blot appears slightly above $70 \mathrm{kDa}$ and the NA band shows around $55 \mathrm{kDa} t$ which leads to the conclusion that the HA and NA are glycosylated. Insect cells perform complex glycosylation but in a slightly different manner than mammalian cells. Nevertheless, any negative effect resulted from different pattern of glycosylation on the immunogenicity of the recombinant HA and NA has not yet been reported in the in vivo studies.

Taken together, the recombinant baculovirus constructed in this study has proper characteristics that can generate H5N1 influenza VLP in Sf9 cells for vaccine purpose. Experiments are in progress to produce large quantity of purified $\mathrm{H} 5 \mathrm{~N} 1$ influenza complex proteins to prove formation of VLP in electromicroscopy and assay its immunogenicity in mouse model. 


\section{Acknowledgements}

The authors would like to thank Mr. Hadi Lashini for logistical assistance.

\section{Authors' Contribution}

All authors have contributed equally.

\section{Financial Disclosure}

There is no Financial Disclosure.

\section{Funding/Support}

This work was supported by Medical Research Center of Baqiyatallah University.

\section{References}

1. Munster VJ, Fouchier RA. Avian influenza virus: of virus and bird ecology. Vaccine. 2009;27(45):6340-4.

2. Alexander Dennis J. A review of avian influenza in different bird species.Vet microbiol 2000;74(1):3-13.

3. Knipe DM, Howley P. Fields Virology:: Wolters Kluwer Health; 2013.

4. Wen Z, Ye L, Gao Y, Pan L, Dong K, Bu Z, et al. Immunization by influenza virus-like particles protects aged mice against lethal influenza virus challenge. Antiviral Res. 2009;84(3):215-24.

5. Hitchman RB, Possee RD, Crombie AT, Chambers A, Ho K, Siaterli E, et al. Genetic modification of a baculovirus vector for increased expression in insect cells. Cell Biol Toxicol. 2010;26(1):57-68.

6. Ligon BL. Avian influenza virus H5N1: a review of its history and information regarding its potential to cause the next pandemic. Semin Pediatr Infect Dis. 2005;16(4):326-35.

7. Ellebedy AH, Webby RJ. Influenza vaccines. Vaccine. 2009;27 Suppl 4:D65-8.

8. Steel J. New strategies for the development of H5N1 subtype influenza vaccines: progress and challenges. BioDrugs. 2011;25(5):28598.

9. Lambert Linda C, Fauci Anthony S. Influenza vaccines for the future. New England J Med. 2010;363(21):2036-2044.

10. Berger I, Fitzgerald DJ, Richmond TJ. Baculovirus expression system for heterologous multiprotein complexes. Nat Biotechnol. 2004;22(12):1583-7.

11. Bright RA, Carter DM, Daniluk S, Toapanta FR, Ahmad A, Gavrilov $\mathrm{V}$, et al. Influenza virus-like particles elicit broader immune responses than whole virion inactivated influenza virus or recombinant hemagglutinin. Vaccine. 2007;25(19):3871-8.
12. Kang SM, Pushko P, Bright RA, Smith G, Compans RW. Influenza virus-like particles as pandemic vaccines. Curr Top Microbiol Immunol. 2009;333:269-89.

13. Ludwig C, Wagner R. Virus-like particles-universal molecular toolboxes. Curr Opin Biotechnol. 2007;18(6):537-45.

14. Krammer F, Grabherr R. Alternative influenza vaccines made by insect cells. Trends Mol Med. 2010;16(7):313-20.

15. Ausubel FM, Brent R, Kingston RE, Moore DD, Seidman JG, Smith JA, et al. Short Protocols in Molecular Biology: Wiley; 2002.

16. SambrookJ, Fritsch EF, Maniatis T. Molecular Cloning: A Laboratory Manual.: Cold Spring Harbor laboratory Press; 1989.

17. Aucoin MG, Mena JA, Kamen AA. Bioprocessing of baculovirus vectors: a review. Curr Gene Ther. 2010;10(3):174-86.

18. Trowitzsch S, Bieniossek C, Nie Y, Garzoni F, Berger I. New baculovirus expression tools for recombinant protein complex production. J Struct Biol. 2010;172(1):45-54.

19. Federico Maurizio. Virus-like particles show promise as candidates for new vaccine strategies. Future virology. 2010;5(4):371-374.

20. Latham T, Galarza JM. Formation of wild-type and chimeric influenza virus-like particles following simultaneous expression of only four structural proteins. J Virol. 2001;75(13):6154-65.

21. Galarza JM, Latham T, Cupo A. Virus-like particle (VLP) vaccine conferred complete protection against a lethal influenza virus challenge. Viral Immunol. 2005;18(1):244-51.

22. Bright RA, Carter DM, Crevar CJ, Toapanta FR, Steckbeck JD, Cole $\mathrm{KS}$, et al. Cross-clade protective immune responses to influenza viruses with H5N1 HA and NA elicited by an influenza virus-like particle. PLoS One. 2008;3(1).

23. Quan FS, Steinhauer D, Huang C, Ross TM, Compans RW, Kang SM. A bivalent influenza VLP vaccine confers complete inhibition of virus replication in lungs. Vaccine. 2008;26(26):3352-61.

24. Sokolenko S, George S, Wagner A, Tuladhar A, Andrich JM, Aucoin MG. Co-expression vs. co-infection using baculovirus expression vectors in insect cell culture: Benefits and drawbacks. Biotechnol Adv. 2012;30(3):766-81.

25. Quan FS, Huang C, Compans RW, Kang SM. Virus-like particle vaccine induces protective immunity against homologous and heterologous strains of influenza virus. JVirol. 2007;81(7):3514-24.

26. Kang SM, Yoo DG, Lipatov AS, Song JM, Davis CT, Quan FS, et al. Induction of long-term protective immune responses by influenza H5N1 virus-like particles. PLoS One. 2009;4(3).

27. Pushko P, Tumpey TM, Bu F, Knell J, Robinson R, Smith G. Influenza virus-like particles comprised of the HA, NA, and M1 proteins of H9N2 influenza virus induce protective immune responses in BALB/c mice. Vaccine. 2005;23(50):5751-9.

28. Pushko P, Tumpey TM, Van Hoeven N, Belser JA, Robinson R, Nathan $\mathrm{M}$, et al. Evaluation of influenza virus-like particles and Novasome adjuvant as candidate vaccine for avian influenza. Vaccine. 2007;25(21):4283-90. 